\title{
Investigation of the efficiency of a high temperature heat storage charging
}

\author{
Artur Nemś ${ }^{1, a}$, Agata Hołowczak ${ }^{1}$ and Magdalena Nemś ${ }^{1}$ \\ ${ }^{1}$ Wroclaw University of Technology, Faculty of Mechanical and Power Engineering, Poland
}

\begin{abstract}
The article presents the results of the test of a heat storage filled with chamotte brick. The air parameters were used as the basis for determining the heat stream represented by a working medium, which was then compared with the stream accumulated in the material filling the deposit. Additionally, heat streams were shown for individual segments of the deposit. The efficiency of heat accumulation was determined for each of them. They showed that the efficiency of accumulation process was highest in the deposit's first segments and the lowest in the last segments, where the air was the coldest. The dynamics of the efficiency's shift was different for each of the observed segments. In the area closest to the inlet of hot air, the dynamics of the process of heat accumulation decreased the fastest and after one hour only a slight increase of efficiency was visible in the function of time. In the accumulation material placed closest to the outlet, the process of absorbing heat was very fast, starting from the 20th minute before the tests were completed. The smallest changes in the process' character, throughout the whole time of testing, were recorded in the middle layer.
\end{abstract}

\section{Introduction}

Accumulation of heat is a very important issue, especially when there is a periodically available source of waste heat. Sensible heat storage materials are rocks, minerals, water, oil and different kinds of composite materials. Their alternative is accumulation in phase-change materials or materials that use chemical reactions. Apart from the ability to work in a broad range of temperatures, sensible materials have many advantages, such as: durability and low requirements as for the process' conditions, as well as for the heat storage's construction itself. Working media in such systems are usually gases, most often air.

There are studies devoted to accumulation in sensible heat storage materials. The following have been considered: the influence of shape on heat transfer [1], pressure drops [2], and optimisation of the process of charging and discharging [3, 4]. The most popular practical application is using them in connection with solar installations. Low-temperature rock storages are used in warm climate countries, where in combination with flat solar collectors they level the temperature in residential buildings [5]. Also natural rock caves are used as storages, being seasonal heat accumulators for whole housing estates, and they are charged during summer [6]. Hightemperature installations are used in advanced systems, usually with concentrating solar collectors. They can be found in solar power plants as well as in heating systems still in the test phase $[7,8]$. Also, a combination with high-temperature waste heat from technological processes and with air fireplaces using biomass is possible.

Further part of the article presents experimental tests of the efficiency of charging a heat storage filled with chamotte bricks. The installation is at the research stage and its purpose is cooperation with air system such as solar concentrating collector or the aforementioned biomass fireplace.

\section{Set-up}

The set-up shown in Fig. 1 is made of cuboidal sheet metal housings, having between them insulator in the form of mineral wool, 15-centimetre thick on vertical walls and 30-centimetre thick on horizontal walls. Internal housing is filled with accumulating material. The installation works in the following way: a ventilator sucks in ambient air, which flows from the inlet channel through the gas-meter, to the storage. Then, the air is heated until it reaches the desired temperature. Inside the deposit, the air transfers the heat to the material filling the storage and the deposit is charged. Cold working medium is taken out through the outlet channel. The system works in an open cycle.

Fig. 2 shows the measuring set-up during conducted tests. Air inlet at the bottom of the smaller housing is placed under metal mesh, on which chamotte plates were put. Such a solutions allows for an even charging of the deposit by distributing the air over the whole surface of the filling.

\footnotetext{
${ }^{\mathrm{a}}$ Corresponding author: artur.nems@pwr.edu.pl
} 


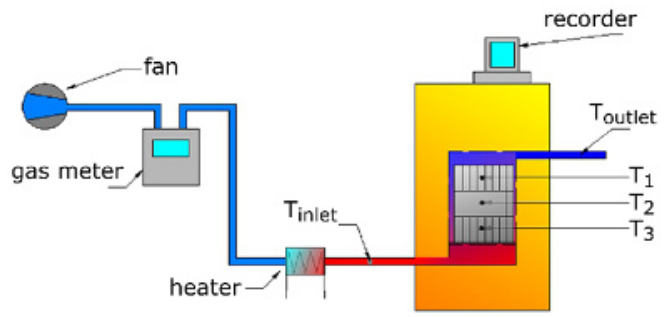

Figure 1. Measuring set-up marked with temperature taking points and with measuring equipment.
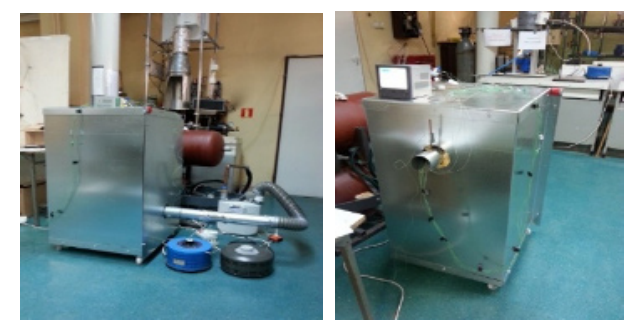

Figure 2. Research set-up. Air inlet together with gas-meter, autotransformer and ventilator visible on the right, outlet and measurements recorder on the left.

The deposit was filled with KNAP chamotte bricks with specific heat of $0.84 \mathrm{~kJ} / \mathrm{kg} \cdot \mathrm{K}$ and single brick mass of $1.74 \mathrm{~kg}$. The accumulating material was laid alternately in three layers, as shown on Fig. 3 and 4.
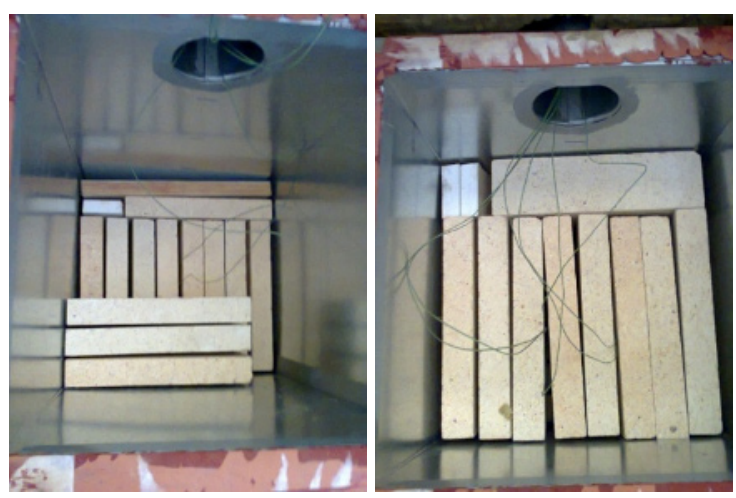

Figure 3. Layout of material filling the storage.

Fig. 4 shows the layout of plates in the storage in each of the three segments. The number of bricks is different and it is 9, 7 and 9 pieces on the lower, middle and upper level respectively. Also, empty spaces without filling material are visible. They result from the specific size of the bricks, making it impossible to fill the storage accurately. Those places were filled with different material, visible on Fig. 3, characterised by low heat capacity. Such a solution allows for an even distribution of air in the storage and low thermal conductivity coefficient allows to omit those elements in further calculations due to their insignificant influence on the process.

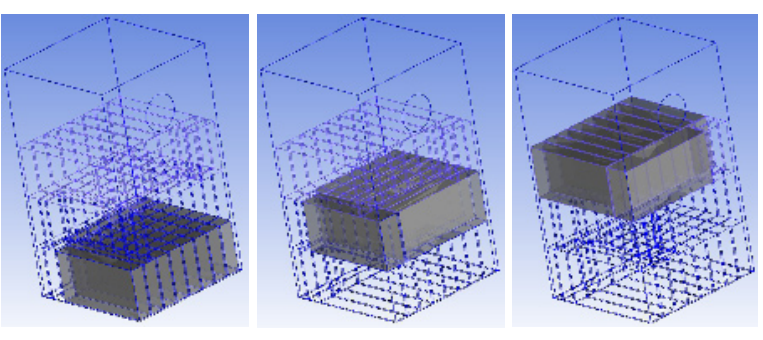

Figure 4. Chamotte brick layout in the storage on the lower, middle and upper level.

The deposit's purpose is accumulation of thermal energy supplied, for instance, by a solar collector. In order to determine the amount of heat possible to be accumulated, the measurements of temperature and volume of air were conducted in laboratory conditions. The volume was measured with ACD-G16C gas-meter, initially every 5, 10, and then every 30 minutes. Thermocouple readings were recorded with a 24-channel KD7 screen recorder. The main aim during the research was the determination of the storage's charging characteristics. Thermocouples distributed according to the scheme shown in Fig. 1 allowed for the recording of temperature, at one-minute intervals, of the air at the inlet and outlet, as well as of the accumulating material, in each of the 3 layers. The study of the charging process lasted for 2 hours and 44 minutes.

In the examined storage, the following assumptions were accepted in order to describe the heat transfer:

- stability of the physical properties of the accumulating material (bricks) and the heating medium (air),

- stability of the air temperature at the inlet of the storing system in the whole section,

- stability of the air speed in the deposit's cross-section and its invariability along its length,

- not taking into account the heat transfer along the air flow direction [9].

\section{Analysis of the results}

The following values were obtained as the result of conducted tests:

- ambient temperature: $t_{a m b}=18^{\circ} \mathrm{C}$,

- volume of the flowing air: $\dot{V}_{\text {avg }}=0.00433 \frac{\mathrm{m}^{3}}{\mathrm{~s}}$,

- temperatures in different areas of the storage were put together in Table 1.

The calculations allowed to determine the characteristics of storage charging, actual and maximum amount of accumulated heat and the process' efficiency. It enabled the analysis of high-temperature heat accumulation in the deposit filled with chamotte bricks.

Fig. 5 shows the rise of the storage's temperature during charging in individual segments: lower (T1), middle (T2) and upper (T3). The quickest temperature rise is observed on the lowest lever of the storage, right above the air inlet. It results from the biggest difference between the temperature of air and the deposit. The slowest rise of temperature is observed in the upper part of the storage, reached by air of lower temperature. The air flowing 
through the storage cools down, transferring heat to the material. When analysing the diagram, one can notice that 80 minutes after the beginning of the experiment the increase ratio of temperatures becomes constant, which is shown by means of parallel straight lines on the diagram.

Table 1. Partial results of temperature measurements in different areas of the storage.

\begin{tabular}{|l|c|c|c|c|c|}
\hline$\tau, \min$ & $t_{\text {inlet }},{ }^{\circ} \mathrm{C}$ & $t_{\text {outlet }},{ }^{\circ} \mathrm{C}$ & $t_{1},{ }^{\circ} \mathrm{C}$ & $t_{2},{ }^{\circ} \mathrm{C}$ & $t_{3},{ }^{\circ} \mathrm{C}$ \\
\hline 1 & 61.3 & 18.8 & 17.9 & 18.3 & 18.5 \\
\hline 10 & 155.1 & 26.1 & 20.7 & 24.3 & 19.1 \\
\hline 20 & 159.3 & 29.3 & 29.3 & 30.5 & 20.0 \\
\hline 30 & 159.8 & 33.1 & 39.1 & 36.8 & 21.3 \\
\hline 40 & 160.2 & 36.6 & 48.7 & 42.8 & 23.0 \\
\hline 50 & 160.8 & 39.9 & 57.6 & 49.1 & 25.3 \\
\hline 60 & 160.9 & 42.9 & 65.9 & 55.1 & 27.9 \\
\hline 80 & 161.0 & 47.9 & 80.0 & 66.2 & 30.9 \\
\hline 100 & 161.2 & 55.3 & 91.5 & 76.1 & 34.3 \\
\hline 120 & 161.3 & 62.3 & 100.8 & 85.0 & 37.7 \\
\hline 140 & 161.9 & 68.9 & 108.2 & 93.1 & 41.5 \\
\hline 160 & 161.0 & 75.3 & 114.4 & 100.1 & 45.3 \\
\hline 164 & 160.9 & 76.1 & 115.2 & 101.1 & 49.3 \\
\hline
\end{tabular}

Temperature measured in different parts of the storage enables the determination of the stream of accumulated heat. The most crucial value is the amount of actually accumulated heat, resulting from the material's (chamotte bricks') accumulating capacity. The calculations below allowed to determine the amount of accumulated energy, the actual and maximum stream of heat. This lets us determine the speed of storage's charging and the process' efficiency.

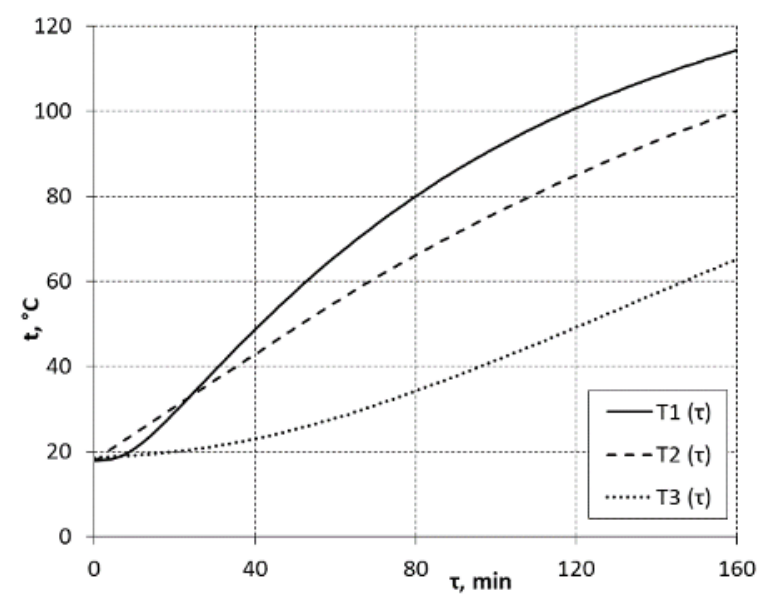

Figure 5. Distribution of temperature in individual segments of the storage in the function of time.

The amount of accumulated energy is described with equation (1).

$$
\begin{gathered}
Q_{z m}=m_{z} \cdot c_{p_{z}} \cdot \Delta t_{\text {avg }}= \\
=m_{z} \cdot c_{p_{z}} \cdot\left(t_{\text {avg } 2}-t_{\text {avg } 0}\right), k J
\end{gathered}
$$

where:

- $m_{z}$ - total mass of chamotte bricks, $\mathrm{kg}$,

- $c_{p_{z}}$ - specific heat of the bricks equal to $0.84 \frac{\mathrm{kJ}}{\mathrm{kg} \cdot \mathrm{K}}$, $-t_{\text {avg } 2}$ - average temperature of the storage at the moment of measurement, ${ }^{\circ} \mathrm{C}$,

- $t_{\text {avg } 0}$ - average temperature of the storage at the beginning of measurements, ${ }^{\circ} \mathrm{C}$.

The average temperature is the temperature averaged in individual layers of the storage (2).

$$
t_{\text {avg }}=\frac{t_{1}+t_{2}+t_{3}}{3},{ }^{\circ} \mathrm{C}
$$

Total mass of the storage $\mathrm{m}_{\mathrm{z}}$ is:

$$
m_{z}=m_{z 1} \cdot n, k g
$$

where:

- $m_{z 1}-$ single brick mass equal to $1.74 \mathrm{~kg}$,

- $n$ - number of bricks.

The increase of the accumulated air in the storage is shown in Fig. 6.

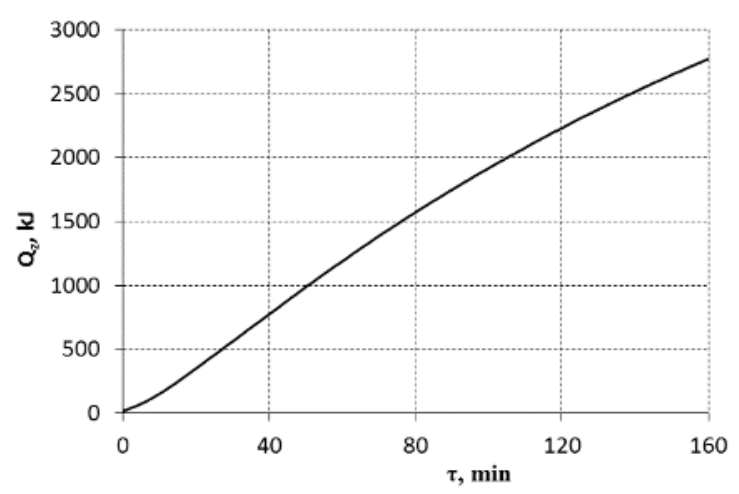

Figure 6. The amount of thermal energy accumulated in the storage.

On the basis of the obtained results, one can see an almost linear relationship between the amount of accumulated thermal energy and the time of the process' duration. The temperature keeps rising during the experiment, which directly influences the increase of accumulated heat.

Maximum heat stream possible to be accumulated in the storage is equal to the stream of heat carried by heated air. The air flowing through the storage cools down and gives its energy to chamottle bricks. The amount that the gas can transfer was determined on the basis of equation 4. Treating air as ideal gas, the following were assumed [10]:

- density: $\rho_{\text {air }}=1.2 \frac{\mathrm{kg}}{\mathrm{m}^{3}}$,

- specific heat: $c_{p_{\text {air }}}=1.005 \frac{\mathrm{kJ}}{\mathrm{kg} \cdot \mathrm{K}}$.

$$
\begin{gathered}
\dot{Q}_{\text {max }}=\dot{m}_{\text {air }} \cdot c_{p_{\text {air }}} \cdot \Delta t= \\
=\dot{V}_{\text {air }} \cdot \rho_{\text {air }} \cdot c_{p_{\text {air }}} \cdot\left(t_{\text {inlet }}-t_{\text {outlet }}\right), k J
\end{gathered}
$$

where:

- $t_{\text {inlet }}$ - temperature of air at the inlet, ${ }^{\circ} \mathrm{C}$,

- $t_{\text {outlet }}$ - temperature of air at the outlet, ${ }^{\circ} \mathrm{C}$.

Fig. 7 shows calculation results of maximum heat stream possible to be transferred by flowing air. 


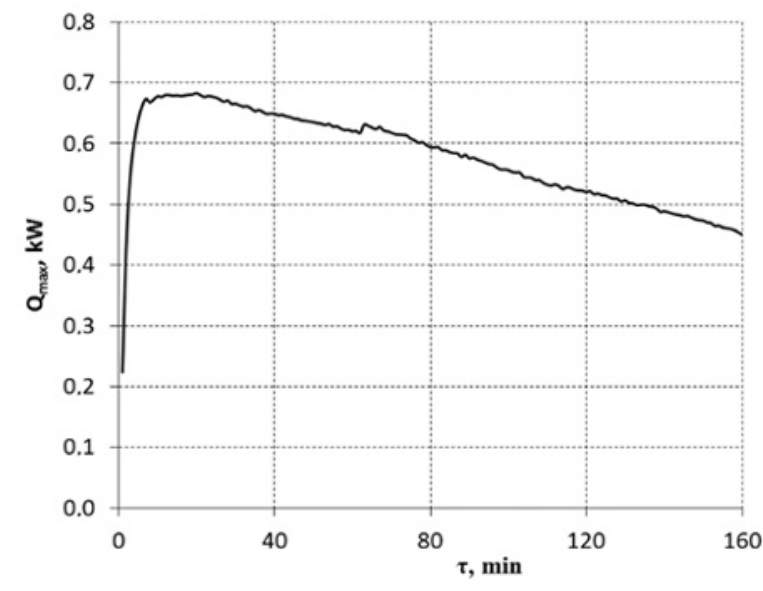

Figure 7. Characteristics of the maximum heat possible to be accumulated in the function of time.

When analysing the results, one can notice a low maximum value of the heat stream in the first minutes of the measurements. The reason behind this may be the storage's temperature equal to ambient temperature. Unheated set-up (cool metal housings) absorbed large amounts of heat from the flowing air at the beginning. However, as the storage is further charged, the value of maximum heat stream decreases. It's main reason is the growing heat loss (from the material to the surroundings) as chamotte bricks are being heated. It results in a lower drop of the temperature of air flowing through the storage. It leads to a decreasing difference of temperatures between the inlet air (relatively constant value) and the outlet air (rising with the duration of the experiment), which directly influences the decrease of the value of maximum heat possible to be accumulated. The stream of accumulated air was determined on the basis of equation 5 and it is shown in Fig. 8.

$$
\dot{Q}_{z m}=\frac{m_{z} \cdot c_{p_{Z}} \cdot \Delta t_{\text {avg }}}{\tau}=\frac{m_{z} \cdot c_{p_{Z}} \cdot\left(t_{\text {avg } 2}-t_{\text {avg } 0}\right)}{\tau}, k W
$$

where:

- $\tau$-duration of the experiment, $\mathrm{s}$.

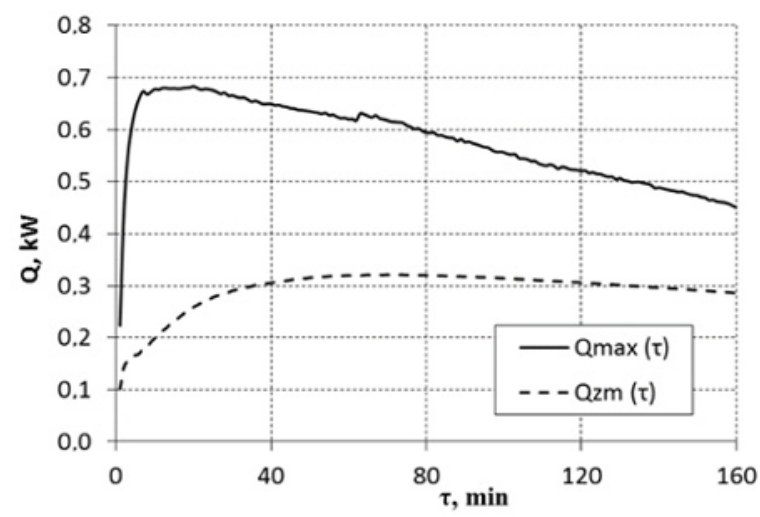

Figure 8. Characteristics of the maximum and actual heat in the function of time.

Fig. 8 shows the increase of the stream of actually accumulated heat in the function of time. It is caused by growing temperature of the storage, and by increasing temperature difference determined on the basis of equation 5. After 73 minutes of the measurements, value $\dot{Q}_{z m}$ slightly decreases and the process of storage charging is slower from that moment on. It results from the decreasing relationship between temperature difference and duration of the test, which in turn can be caused by greater losses to the surroundings. When comparing both characteristics, it can be seen that the value of maximum heat stream decreases with the increase of the value of actual stream. The diagram allows us to draw the conclusion that the difference between these values is levelled with time. The rising temperature of the storage with a stable temperature of inlet air cause an increase of heat losses from chamotte bricks to the surroundings, which directly leads to the rise of outlet temperature.

In order to picture the process of the storage's charging, the stream of accumulated heat in each of the deposit's segments was calculated, according to equation 5. In this case, the mass of filling material was calculated on the basis of equation 3 , only the number of plates is different:

- on level 1 (lower): $\mathrm{n}=9$,

- on level 2 (middle): $\mathrm{n}=7$,

- on level 3 (upper): $n=9$.

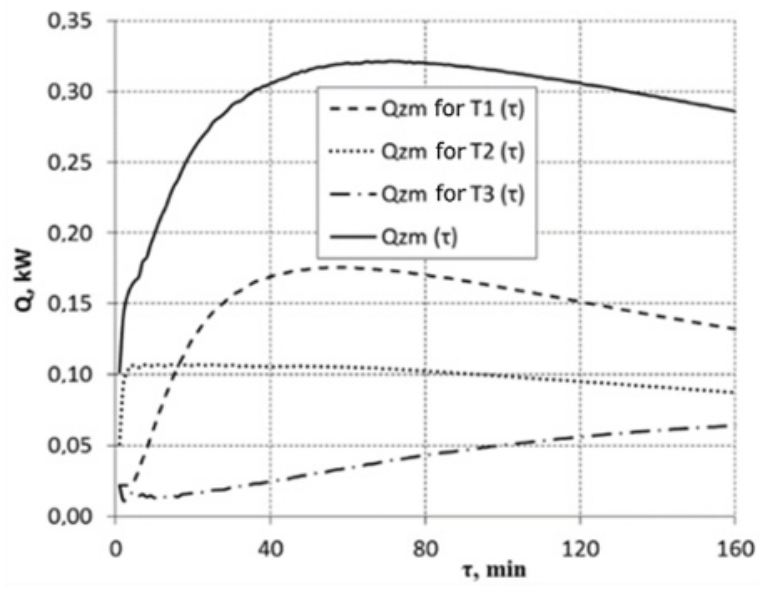

Figure 9. Relationship between the stream of accumulated air and the time for individual segments of the storage.

Calculation results are shown in Fig. 9. The characteristics for the lower part of the storage is analogous to the diagram of the total stream of accumulated heat (Fig. 7). Bot characteristics show a quick increasing tendency during the first hour of the process. After approx. $60 \mathrm{~min}$. value $\dot{Q}_{z m}$ for the lowest level of the storage starts to decrease. In the middle segment, value $\dot{Q}_{z m}$ remains almost on an even level. The lowest value of the stream of accumulated heat is observed in the upper segment, but differently from other layers, there is a growing tendency there. It is worth stressing that values $\dot{Q}_{z m}$ for individual layers become more similar to one another with time. Thus, it can be concluded that the longer the process of storage charging, the more level is the distribution of accumulated heat in its layers. 
The obtained characteristics result from temperature characteristics of the deposit - the higher the temperature of the deposit, the higher the value $\dot{Q}_{z m}$. The sum of three characteristics of individual segments equals the total value of actual heat stream.

Knowing the maximum and actual stream of accumulated air, we can determine the process' efficiency (equation 6) being the relationship between actually accumulated heat and the maximum heat determined on the basis of equation 4 . The characteristics of the efficiency of storage charging is shown in Fig. 10.

$$
\varepsilon=\frac{\dot{Q}_{z m}}{\dot{Q}_{\max }}
$$

The efficiency of the process of charging increases with the time of the experiment's duration. On the basis of the diagram's shape it is possible to conclude that the increase of efficiency is logarithmic. It means that with time the charging process will stabilise, which results from the gradual levelling of the values of maximum and accumulated heat stream. Final efficiency in the conducted experiment is on the level of over $60 \%$. The curve shows a clear relationship between the efficiency value and the time of the process. Theoretically, in infinitely long time, this coefficient would be $100 \%$.

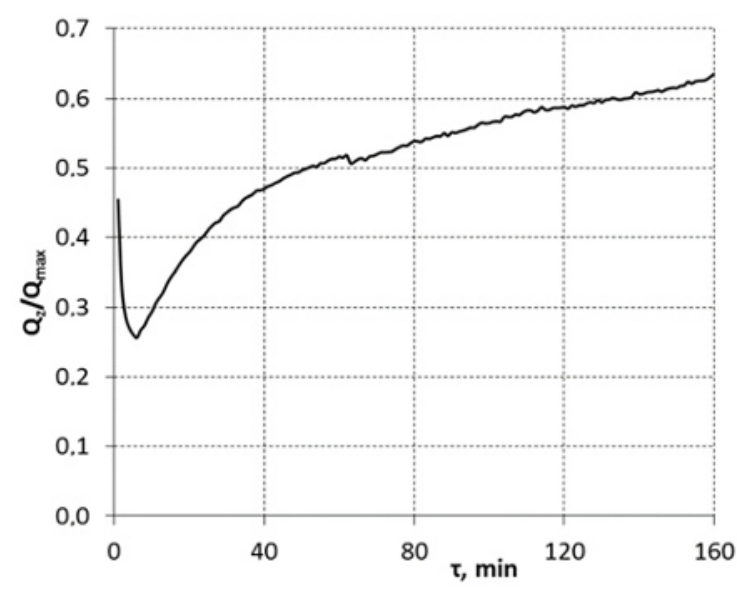

Figure 10. Diagram of the efficiency of charging process in time.

The efficiency for individual segments was determined on the basis of equation 6. The value of actually accumulated energy $\dot{Q}_{z m i}$ was assumed as the accumulated heat and the value of maximum heat stream for the whole storage was assumed as $\dot{Q}_{\max }$. The results are shown in Table 2.

Table 2. Partial results of partial calculations of the process' charging efficiency.

\begin{tabular}{|c|c|c|c|c|c|c|c|c|c|c|}
\hline$\tau, \min$ & $\dot{Q}_{z m}, \mathrm{~kJ}$ & $\dot{Q}_{\text {max }}, \mathrm{kW}$ & $\dot{Q}_{z m}, \mathrm{~kW}$ & $\varepsilon,-$ & $\dot{Q}_{z m 1}, \mathrm{~kW}$ & $\dot{Q}_{z m 2}, \mathrm{~kW}$ & $\dot{Q}_{z m 3}, \mathrm{~kW}$ & $\varepsilon_{1},-$ & $\varepsilon_{2,}-$ & $\varepsilon_{3,-}$ \\
\hline 10 & 119 & 0.678 & 0.199 & 0.294 & 0.064 & 0.107 & 0.013 & 0.094 & 0.158 & 0.019 \\
\hline 20 & 311 & 0.683 & 0.259 & 0.379 & 0.126 & 0.107 & 0.016 & 0.185 & 0.156 & 0.024 \\
\hline 30 & 523 & 0.666 & 0.290 & 0.436 & 0.156 & 0.107 & 0.020 & 0.234 & 0.161 & 0.031 \\
\hline 40 & 733 & 0.649 & 0.306 & 0.470 & 0.169 & 0.106 & 0.025 & 0.261 & 0.163 & 0.038 \\
\hline 50 & 946 & 0.635 & 0.315 & 0.497 & 0.175 & 0.106 & 0.030 & 0.275 & 0.167 & 0.047 \\
\hline 60 & 1152 & 0.620 & 0.320 & 0.516 & 0.176 & 0.105 & 0.034 & 0.283 & 0.170 & 0.055 \\
\hline 70 & 1348 & 0.618 & 0.321 & 0.520 & 0.174 & 0.104 & 0.039 & 0.281 & 0.169 & 0.063 \\
\hline 80 & 1537 & 0.594 & 0.320 & 0.539 & 0.170 & 0.103 & 0.043 & 0.287 & 0.173 & 0.073 \\
\hline 90 & 1714 & 0.576 & 0.317 & 0.551 & 0.166 & 0.101 & 0.047 & 0.289 & 0.175 & 0.081 \\
\hline 100 & 1885 & 0.556 & 0.314 & 0.565 & 0.162 & 0.099 & 0.050 & 0.290 & 0.178 & 0.091 \\
\hline 110 & 2046 & 0.532 & 0.310 & 0.582 & 0.157 & 0.097 & 0.053 & 0.294 & 0.182 & 0.100 \\
\hline 120 & 2202 & 0.520 & 0.306 & 0.588 & 0.152 & 0.095 & 0.056 & 0.292 & 0.183 & 0.108 \\
\hline 130 & 2347 & 0.507 & 0.301 & 0.594 & 0.146 & 0.093 & 0.059 & 0.289 & 0.184 & 0.116 \\
\hline 140 & 2488 & 0.489 & 0.296 & 0.606 & 0.142 & 0.091 & 0.061 & 0.290 & 0.187 & 0.124 \\
\hline 150 & 2620 & 0.473 & 0.291 & 0.615 & 0.137 & 0.089 & 0.063 & 0.289 & 0.189 & 0.132 \\
\hline 160 & 2745 & 0.450 & 0.286 & 0.635 & 0.132 & 0.087 & 0.064 & 0.294 & 0.194 & 0.142 \\
\hline 164 & 2781 & 0.446 & 0.283 & 0.634 & 0.130 & 0.086 & 0.064 & 0.292 & 0.194 & 0.143 \\
\hline
\end{tabular}




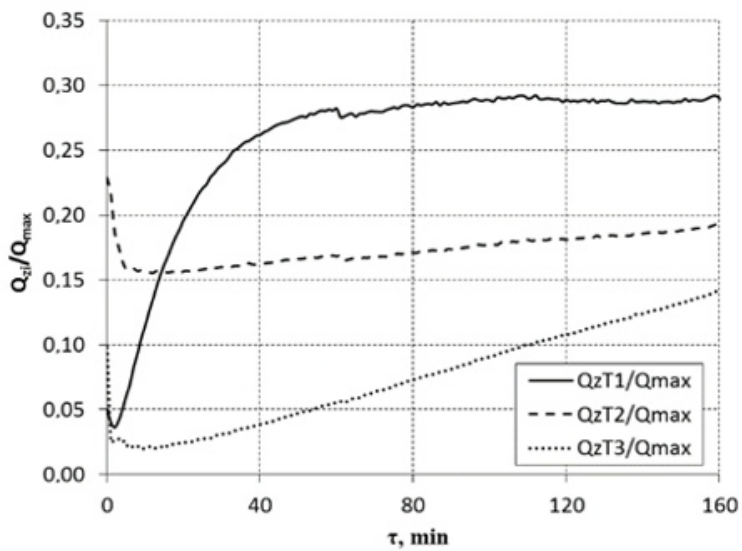

Figure 11. Diagram of the efficiency of the storage's charging in individual segments in the function of time.

As can be concluded on the basis of Fig. 9 and 11, both in the case of the characteristics of accumulated heat and of the charging efficiency, the highest increase is observed for the lowest segment of the deposit. The increase of efficiency for this layer shows a logarithmic character reaching the determined value on the level of less than $30 \%$ as early as after one hour. In the middle segment the efficiency slightly rises, because it increases by less than 5\% during the whole experiment. A relatively high linear increase can be observed for the storage's upper segment. The main reason behind it is a slight increase of accumulated heat accompanied by the decrease of maximum heat stream as the process continued.

\section{Conclusions}

The increase of temperature in the storage influences the rising value of the stream of actually accumulated heat. Analysing the amount of energy accumulated in individual segments of the storage it can be concluded that the longer the process of storage charging, the more level is the distribution of accumulated heat in its layers. The first - lowest level of the storage absorbs the greatest amount of energy.

The efficiency of the process of charging increases with the time of the experiment's duration. It signifies an increase of the heat absorbed by accumulating material from the air. Final efficiency in the conducted experiment is on the level of approx. $60 \%$. In individual segments the highest efficiency is observed for the storage's lowest segment.

The research presented can be treated as an introduction to the analysis of different materials filling the deposit and their influence on the amount of accumulated heat. In the actual installation, the amount of accumulated energy will be directly related to the potential of the heat source supplying the storage. However, it seems that the ability to adjust work parameters at the laboratory set-up are sufficient to simulate actual conditions and can be a major input in the further research of the process of heat accumulation.

\section{References}

1. R. Singh, R.P. Saini, J.S. Saini, Sol. Energy 80, (2006)

2. K. Sagara, N. Nakahara, Sol. Energy 47, 3 (1991)

3. N.G. Barton, Appl. Therm. Eng. 55, (2013)

4. A. Mawire, M. McPherson, R.R.J. van den Heetkamp, S.J.P. Mlatho, Appl. Energy 86, (2009)

5. I.A. Abbud, G.O.G. Lof, D.C.Hittle, Sol. Energy, 54, 2 (1995)

6. J. Xu, R.Z. Wang, Y. Li, Sol. Energy 103, (2014)

7. H.B. Madessa, T. Veslum, J. Løvseth, O.J. Nydal, 30th ISES Biennial Solar World Congress 2011, SWC 20113 (2011)

8. M. Nemś, J. Kasperski, Energy Procedia 57, (2014)

9. R. Domański, Magazynowanie energii cieplnej (PWN, Warszawa, 1990)

10. E. Kalinowski, Przekazywanie ciepta $i$ wymienniki (Oficyna Wydawnicza Politechniki Wrocławskiej, Wrocław, 1995) 